\title{
High Energy Neutrino expectations from the Central Molecular Zone
}

\author{
Antonio Marinelli*† \\ INFN - Sezione di Pisa and Dipartimento di Fisica dell' Universitá di Pisa, Largo B. Pontecorvo \\ 3, 56127 Pisa, Italy \\ E-mail: antonio.marinelli@pi.infn.it
}

\section{Daniele Gaggero}

GRAPPA, University of Amsterdam, Science Park 904, 1098 XH Amsterdam, Netherlands

E-mail: D.Gaggero@uva.nl

\section{Dario Grasso}

INFN - Sezione di Pisa and Dipartimento di Fisica dell' Universitá di Pisa, Largo B. Pontecorvo 3, 56127 Pisa, Italy

E-mail: dario.grasso@pi.infn.it

\section{Marco Taoso}

Instituto de Física Teórica (IFT), UAM/CSIC, Cantoblanco, Madrid, Spain

E-mail: m.taosodcsic.es

\section{Alfredo Urbano}

CERN, Theoretical Physics Department, Geneva, Switzerland

E-mail: alfredo.leonardo.urbanodcern.ch

\section{Sofia Ventura}

INFN - Sezione di Pisa and Dipartimento di Fisica dell' Universitá di Pisa, Largo B. Pontecorvo 3, 56127 Pisa, Italy E-mail: sofia.ventura@pi.infn.it

The density of molecular gas in the central few hundreds parsecs of our Galaxy is estimated to be two orders of magnitude larger than the Galactic average one. This, so called, Central Molecular Zone, represent a privileged target where to look for a signature of Galactic cosmic ray interactions. Here we report the diffuse neutrino expectations from this region following a recently introduced phenomenological scenario. This model reproduces the Fermi-LAT and H.E.S.S. observations of the central 200 Parsecs without the need of local cosmic-ray accelerators. Once fixed the gas and source distribution parameters to reproduce the normalization of the gammaray observed, we use the same framework to obtain the neutrino emission from the molecular clouds Sagittarius B and Sagittarius A and for the region of the inner 200 parsecs. We compare our expectations with the point-like sensitivity of IceCube for this region of the sky and we consider also possible point-like contributions due to Supernovae Remnants contained inside this region. Finally we discuss the future perspectives of the Global Neutrino Network to characterize the neutrino emission from Central Molecular Zone and the possibility to determine the Galactic cosmic-ray cutoff through the IceCube HESE events.

35th International Cosmic Ray Conference - ICRC2017

10-20 July, 2017

Bexco, Busan, Korea 


\section{Introduction}

With Central Molecular Zone (CMZ) we indicate the region extending few hundred parsecs about the Galactic center (GC) along the Galacic plane (GP). Due to the extreme conditions of star formation rate and gas density, with values greater than $10^{4} \mathrm{~cm}^{-3}$ [1], this region represents a unique target for the Galactic cosmic rays (CRs) [2] and consequently a guaranteed neutrino factory [3]. A gamma-ray emission is also expected due to the CRs interaction and the dedicated observations can be used to constrain the parameters of the CR population and of the gas distribution in this interesting region of the Galaxy. In this work we compute the neutrino flux and signal expectations using a recently introduced phenomenological model (Gamma model) based on a spatially variable cosmic-ray transport along the Galactic radius and tuned on a large Galactic scale to explain the measured diffuse gamma-ray emission [4, 5]. Recently the H.E.S.S. collaboration published new measurements for a ridge of 200 parsecs symmetric respect to the Galactic center (GC) using 250 hours of data [6]. With this new sample of events the H.E.S.S. collaboration obtained a detailed morphology of this region and its total gamma-ray spectral energy distribution (SED). The measured SED resulted harder than the cosmic-ray SED measured at the Earth [7, 8] and in a good agreement with the corresponding SED obtained with Fermi-LAT data at lower energies [2]. While the H.E.S.S. collaboration interpret this hard SED as a consequence of freshly accelerated cosmic rays produced by a local "Pevatron" [9], according to the Gamma model this emission is produced by the cosmic-ray sea present in this region of the Galactic plane interacting with the dense gas [10]. As showed by [2] the Gamma model well explain also the gamma-ray SEDs of individual molecular clouds inside the CMZ like the Sagittarius B and the "pacman" region observed around Sagittarius A* [10]. We start our analysis by setting two terms used in the Gamma framework: the source and the gas distributions. We fix the gas distribution using a 3D model with a normalization of $X_{C O}$ able to match the gas column density map adopted by H.E.S.S. in [9]. Successively we select the source term distribution able to describe the total gamma-ray spectrum obtained for the region $|l|<1.0^{\circ}$ and $|b|<0.3^{\circ}$, considering the PASS 8 data sample [11] of Fermi-LAT and the recent H.E.S.S. data analysis [6]. The selected source distribution description [12] takes into account a complete catalog of supernovae remnants (SNRs) type I e type II resulting in a slightly higher flux of gamma-ray and neutrino produced respect to older source distribution descriptions [13]. With this selected framework for the Gamma model we obtain the expected neutrino SED for the whole 200 parsecs observed as a diffuse gamma-ray region by the H.E.S.S. collaboration and for the contained molecular complexes Sagittarius A ("pacman" region) and B [14]. We report also the expected neutrino spectra following a standard scenario of uniform CR diffusion (Base model) for the whole Galactic plane with the same setting for source and gas distribution. We compare the expected neutrino emissions with the point-like source sensitivity obtained by IceCube collaboration from 7 years of data [15] and with the maximal neutrino flux deduced from a PeV HESE event [16] compatible with this region of the sky. We discuss the perspectives of observation considering the Global Neutrino Network [17] and the possibility to constrain the cutoff of Galactic CRs.

\footnotetext{
${ }^{*}$ Speaker.

${ }^{\dagger}$ A footnote may follow.
} 


\section{Comparison with H.E.S.S. and Fermi-LAT data}

In this section we report the gamma-ray diffuse emission spectrum from $5 \mathrm{GeV}$ up to $50 \mathrm{TeV}$ from the CMZ region determined from the combination of Fermi-LAT and H.E.S.S. data. We extract Fermi-LAT data using the Fermi Science Tools v10r0p5 [18]. We use 470 weeks of PASS 8 data with the event class CLEAN and we apply the recommended quality cuts: (DATA_QUAL==1) $\& \& \quad\left(L A T \_C O N F I G==1\right)$. The exposure is computed using the Fermi-LAT response function P8REP2_CLEAN_V6. Here the data are binned in 20 energy bins equally spaced in log scale between $300 \mathrm{MeV}$ and $300 \mathrm{GeV}$ obtained following [19]. We subtracted the emission due to the point source obtained from the 4-year Point Source Catalog (3FGL) provided by the Fermi-LAT collaboration [20]. The total spectrum measured in the the region $|l|<1.0^{\circ}$ and $|b|<0.3^{\circ}$ between
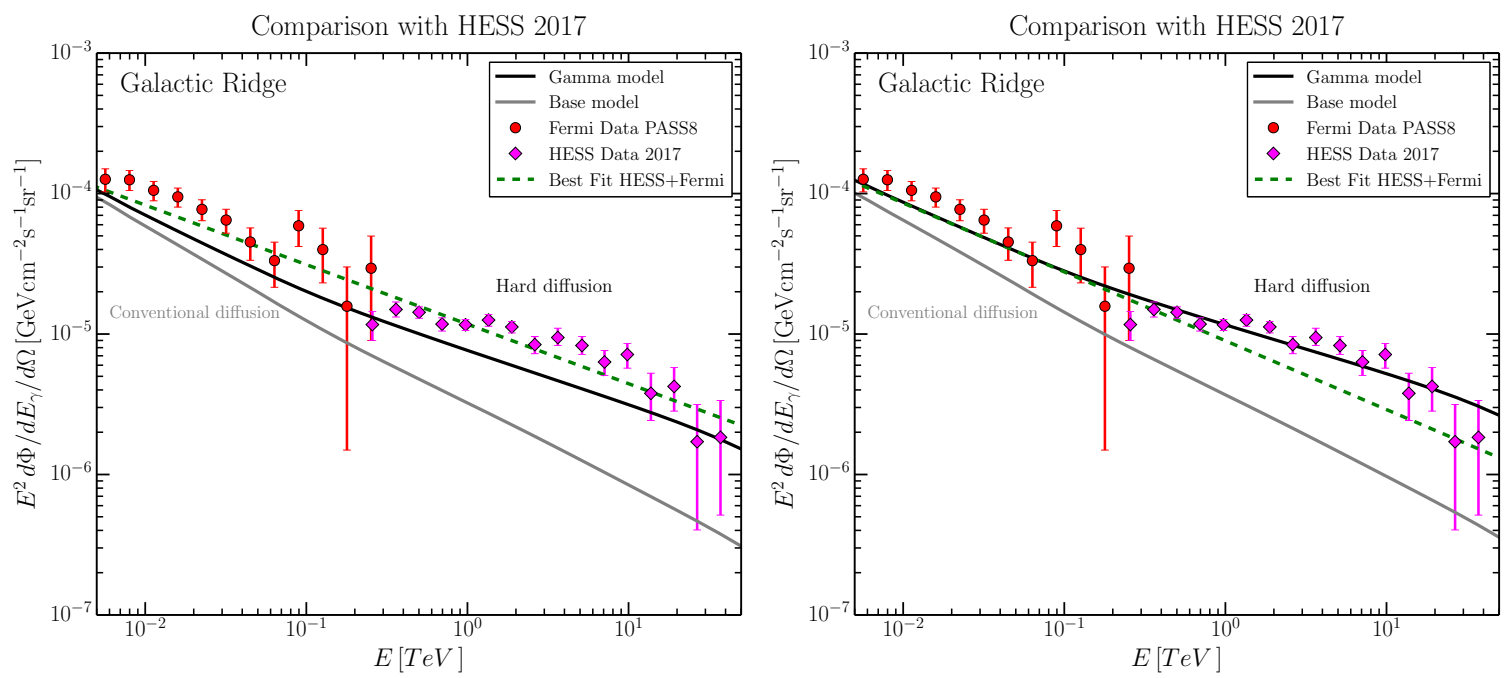

Figure 1: The gamma-ray spectrum in the CMZ region $|l|<1.0^{\circ}$ and $|b|<0.3^{\circ}$. Fermi-LAT data, extracted with the Fermi Science Tools [18] in this work, and H.E.S.S. data from [6] are compared with the contribution of the Gamma and Base models discussed in the text. On the left side the gamma model is obtained considering the source term of [12] and a $X_{C O}=0.4$ while on the right side the Gamma model is obtained considering the source term of [13] and a $X_{C O}=0.6$. The single power-law best fit of the combined data is also reported with the green dashed lines. We have subtracted the contribution of point sources from the FGL catalog released by Fermi-LAT collaboration [20].

$5 \mathrm{GeV}$ and $40 \mathrm{TeV}$ results to be:

$$
\Phi_{G R}=1.181 \times 10^{-5}\left(\frac{E_{\gamma}}{1 T e V}\right)^{-2.42} G e V^{-1} \mathrm{~cm}^{-2} s^{-1} s r^{-1}
$$

and as shown by [2] a similar spectrum is obtained by the regions of Sagittarius $A^{*}$ and Sagittarius B suggesting the same emission process for the whole region. As we can see from the plots reported in Fig. 1 the case of source distribution term [12] can fit the reported GR spectrum without needed of addition components. Considering this result we use the same setup for obtaining the following neutrino expectations. 


\section{Neutrino expectations and comparison with IceCube observations}

In this section we report the expected neutrino SEDs due interaction of the cosmic-ray sea with the gas in the dense central region of our Galaxy. In particular we consider the CMZ region reported by H.E.S.S. $\left(|l|<1.0^{\circ}\right.$ and $|b|<0.3^{\circ}$ ) this year, the "pacman" region (a open ring with $0.15^{\circ}<\theta<0.45^{\circ}$ ) measured around Sagittarius A* reported last year, and an other major cloud present inside the $\mathrm{CMZ}$ observed as a TeV emitter from H.E.S.S. in 2006 [14]: Sagittarius B $\left(|l|<0.5^{\circ}\right.$ and $|b|<0.5^{\circ}$ region reported by H.E.S.S. collaboration). We obtain the expected neutrino SEDs computed with the Gamma model as well as with the Base model, considering two different cases for the cutoffs of primary CRs ( 5 and $50 \mathrm{PeV})$. We show also the recent sensitivity on point-like source searches published by IceCube experiment considering the $v_{\mu}+\overline{v_{\mu}}$ starting track events above $60 \mathrm{TeV}$ collected in 7 years of data tacking. While the "pacman" and the
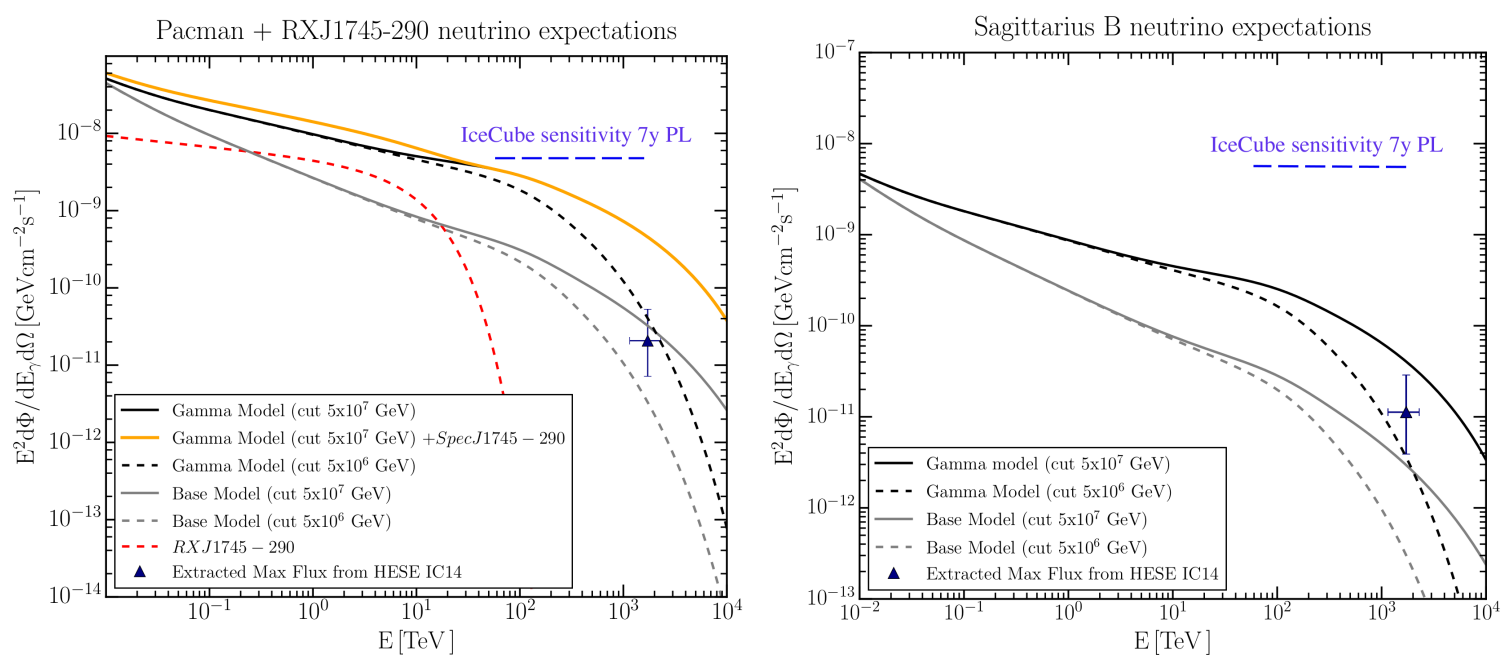

Figure 2: The obtained neutrino SEDs for the molecular clouds Sagittarius A ("pacman" region), on the left side, and Sagittarius B $\left(|l|<0.5^{\circ}\right.$ and $\left.|b|<0.5^{\circ}\right)$, on the right side, considering the introduced Gamma Model and the standard Base Model with two different cutoffs for the primary protons: at 5 and $50 \mathrm{PeV}$ [21]. For the case of the "pacmac" region we reported also a possible neutrino point-like emission of HESS J1745290 extrapolated from the gamma-ray spectrum reported by H.E.S.S. collaboration [14]. Even considering the total gamma-ray flux of HESSJ1745-290 fully hadronic the contribution of neutrino emission inside the "pacman" region is visible only in the low energy range. For comparison we reported also the sensitivity for this region of the sky obtained by IceCube collaboration considering 7 years of data taking with starting track events $\left(v_{\mu}+\overline{v_{\mu}}\right)$ above $60 \mathrm{TeV}$. Reported also the estimation of neutrino flux observed with 4 years of HESE event analysis considering the only PeV neutrino event (IC 14) compatible with the CMZ region.

Sagittarius B molecular clouds are seen as diffuse emitting regions when we observe the gammaray emission with Fermi-LAT and H.E.S.S., with the analysis of the VHE neutrino emission their spatial extensions result compatible with a point-like source search. The CMZ region reported here represent a slightly different case since the spatial extension is on the limit to be considered pointlike emitters, especially for $v_{\mu}+\overline{v_{\mu}}$ analysis. As we can see from Figs. 2 and 3 the sensitivity reached by IceCube with 7 years of starting track events is still not enough to reach the expected flux of Sagittarius B and Sagittarius A molecular clouds when the Gamma model is considered. A different scenario is obtained for the $\mathrm{CMZ}$ region $|l|<1.0^{\circ}$ and $|b|<0.3^{\circ}$ where the sensitivity 
of point-like sources analysis start to be compatible with the expected neutrino flux of Gamma model above $60 \mathrm{TeV}$. It should be noted here that the IceCube sensitivity is calculated for a generic $E^{-2.0}$ spectrum whereas the expected neutrino spectrum from Gamma model follows a power law of $E^{-2.3}$ up to few hundreds of TeV. The total integrated neutrino SED for the CMZ will does not change drastically if we add possible point-like contribution from the recently observed HESS J1746-285 and HESS J1745-290 (in the reported map of Fig. 3 respectively 3FGL J1746-2851C and 2FHL1745.7-2900). This is visible in the plot 2 where the eventual neutrino contribution of the HESS J1745-290 is added to the total spectrum of the "pacman" (Sagittarius A molecular cloud). While the analysis of track-like neutrino events from these regions of the GP will be important to
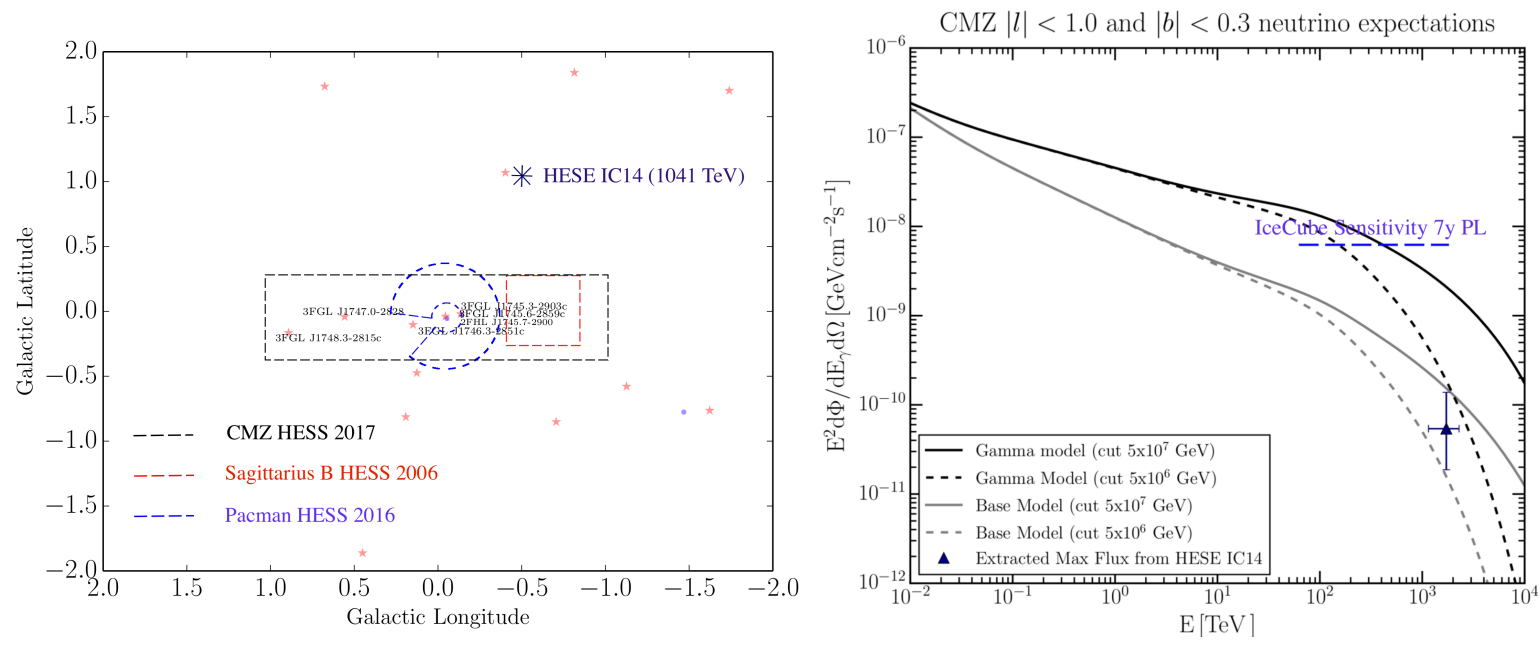

Figure 3: On the left side: the zoomed skymap on the CMZ with the point-like sources seen by Fermi-LAT telescope (with coral stars the 3FGL while with blue violet circle the 2FHL). Possible neutrino point-like sources, observed also as TeV gamma-ray emitters are 3FGL J1746.3-2851c and 2FHL J1745.7-2900. With the navy star we indicate the position of the IceCube HESE shower event IC14 with a reconstructed energy of $1041 \mathrm{TeV}$. Due to its angular incertitude of $13.2^{\circ}$ the entire reported region can be considered compatible with his reconstructed position. With dashed blue, red and black lines we reported the studied regions of Sagittarius A ("pacman"), Sagittarius B (region of H.E.S.S. 2006) and the region of CMZ observed by H.E.S.S. in 2017. The previous two regions are contained in the last one. On the right we report the expected neutrino spectra for Gamma and Base models in comparison to the IceCube point-like sensitivity obtained with 7 years and the flux extrapolated from the HESE event IC14.

constrain the different models of CR sea diffuse emission, with a big improvement expected from ANTARES [22] and future KM3NeT/ARCA [23] observations, the most energetic HESE event collected around the GC by IceCube experiment can give us hints about the cutoff to be applied to the primary CRs. Looking over the 54 HESE events one of them (IC14) with measured energy of $1041_{-144}^{+132} \mathrm{TeV}$ and a angular incertitude of $13.2^{\circ}$ can be spatially related with the position of the CMZ regions considered here. In Figs. 2 and 3 we introduce the maximal neutrino flux computed considering the HESE event compatible with the reported region of the sky in comparison with the neutrino spectra from Gamma and Base models. Since we are referring to a single PeV shower event (IC14, with $13.2^{\circ}$ of angular incertitude) associated with the CMZ region, this flux cannot be 
considered significant to constrain the reported scenarios, however taking into account his energy can give preliminary suggestions about the cutoff of primaries CRs to be used. For all the reported regions (Sagittarius B, "pacman" and CMZ) the obtained flux at PeV seems more compatible with $5 \mathrm{PeV}$ cutoff, however we will have more stringent limits as soon as a more complete HESE catalog will be available.

\section{Results and Conclusions}

In this work we use a recently introduced Gamma model to obtain the expected VHE neutrino emission from the inner 200 Parsecs around the central SMBH of our Galaxy. This model assume an anisotropic diffusion of Galactic CRs along the Galactic radius producing a hardening of the gamma-ray and neutrino spectra when moving through the GC. We start fixing the parameters of source and gas distribution for the Gamma and Base (standard scenario with a uniform CR diffusion along the GP) models. For this purpose we set the $X_{C O}$ able to reproduce the gas column density obtained by H.E.S.S. collaboration for the analysis focused on the GC, and we select the source distribution able to account for the gamma-ray SED recently obtained by the H.E.S.S. collaboration from the region $|l|<1.0^{\circ}$ and $|b|<0.3^{\circ}$. With these setting for the Gamma model we obtain the expected neutrino SEDs from the same region of CMZ observed by H.E.S.S and for the contained molecular clouds of Sagittarius B $\left(0.4^{\circ}<l<0.9^{\circ}\right.$ and $\left.-0.2^{\circ}<b<0.3^{\circ}\right)$ and Sagittarius A (the "pacman" region). We compare the expected neutrino SEDs from Gamma and Base models, taking into account two possible cutoffs for the primary CRs (5 and $50 \mathrm{PeV}$ ), with the point-like source sensitivity obtained by the IceCube collaboration with the analysis of the contained track events $\left(v_{\mu}+\overline{v_{\mu}}\right)$, with energy above $60 \mathrm{TeV}$ reconstructed with 7 years of data taking. As it is shown in Fig. 2 the sensitivity reached for point-like emission by IceCube seems to be not enough for seing the neutrino signal from these two molecular clouds when Gamma model is considered. On the other hand looking at the expected neutrino SEDs integrated over the whole 200 parsecs (Fig. 3) observed recently by H.E.S.S. $\left(|l|<1.0^{\circ}\right.$ and $|b|<0.3 \circ$ ) we obtain more promising expectations with possible positive results on a short time scale. From this region of the sky, a even better sensitivity can be expected from the ANTARES telescope when looking for point-like source through all-flavor analysis [24]. The observation of the CMZ through muonic neutrino channel can be complementary to the analyses focused on a larger portion of Galactic plane, like the one recently published by the ANTARES collaboration (for the region $|l|<40^{\circ}$ and $|b|<3^{\circ}$ ) [22] to understand the emission related to Galactic CRs interaction with the molecular gas. A detailed study of the full CMZ region ( $\leq 600$ parsecs) will became possible in a near future with the incoming KM3NeT/ARCA Mediterranean observatory [25]. Another interesting result to be reached with VHE neutrino observations will be a better constraint on the energy cutoff for the primaries interacting CRs. This estimation cannot be possible for energies above few $\mathrm{PeVs}$ with gamma rays due to the background absorption from distances above few kiloparsecs. For this purpose the neutrino becomes a privileged messenger. Following the scenario introduced with the Gamma model the $\mathrm{CMZ}$ region can be considered a good candidate for producing neutrinos around $\mathrm{PeV}$ energies. In fact 5\% of the total molecular gas present in our Galaxy is contained in a region where we expect also the hardening of diffuse CR SED. However, focusing on the CMZ, this study can be performed exclusively with IceCube events due to the absorption $\mathrm{PeV}$ neutrinos on the Earth before reaching 
the Mediterranean telescopes. From the last catalog of HESE events we can consider one of them (IC14) compatible with the position of the CMZ, giving us the possibility of a first preliminary extrapolation of the maximal neutrino flux at the PeV energy. Even if at the moment this estimation cannot be considered significative due to the angular incertitude of $13.2^{\circ}$ we report here, in Figs. 2 and 3, this exercise for the three selected regions. With the poor statistics of only one HESE event the cutoff of $5 \mathrm{PeV}$ for primary CRs seems to be more compatible with the expected neutrino flux at PeV; however it will be interesting to repeat this study with the future incoming HESE catalogs.

\section{References}

[1] E. A. C. Mills, “The Milky Way's Central Molecular Zone," ArXiv e-prints, May 2017.

[2] D. Grasso et al., "Hard Cosmic Ray Sea in the Galactic Center: a consistent interpretation of H.E.S.S. and Fermi-LAT $\gamma$-ray data," Contribution to ICRC, 2017.

[3] A. Marinelli, D. Gaggero, D. Grasso, M. Taoso, A. Urbano, and M. Valli, "Modeling the Galactic center emission from GeV to PeV," in European Physical Journal Web of Conferences, vol. 136 of European Physical Journal Web of Conferences, p. 03016, Mar. 2017.

[4] D. Gaggero, A. Urbano, M. Valli, and P. Ullio, "Gamma-ray sky points to radial gradients in cosmic-ray transport," Physical Review D, vol. 91, p. 083012, Apr. 2015.

[5] D. Gaggero, D. Grasso, A. Marinelli, A. Urbano, and M. Valli, "The Gamma-Ray and Neutrino Sky: A Consistent Picture of Fermi-LAT, Milagro, and IceCube Results," Astrophysical Journal Letters, vol. 815, p. L25, Dec. 2015.

[6] H. E. S. S. Collaboration, :, H. Abdalla, A. Abramowski, F. Aharonian, F. Ait Benkhali, A. G. Akhperjaniany, T. Andersson, E. O. Angüner, M. Arakawa, and et al., "Characterising the VHE diffuse emission in the central 200 parsecs of our Galaxy with H.E.S.S," ArXiv e-prints, June 2017.

[7] M. Aguilar et al., "Precision Measurement of the Proton Flux in Primary Cosmic Rays from Rigidity $1 \mathrm{GV}$ to $1.8 \mathrm{TV}$ with the Alpha Magnetic Spectrometer on the International Space Station," Phys. Rev. Lett., vol. 114, p. 171103, 2015.

[8] O. Adriani et al., "PAMELA Measurements of Cosmic-ray Proton and Helium Spectra," Science, vol. 332, pp. 69-72, 2011.

[9] A. Abramowski et al., "Acceleration of petaelectronvolt protons in the Galactic Centre," Nature, vol. 531, p. 476, 2016.

[10] D. Gaggero, D. Grasso, A. Marinelli, M. Taoso, and A. Urbano, "Diffuse cosmic rays shining in the Galactic center: A novel interpretation of H.E.S.S. and Fermi-LAT gamma-ray data," ArXiv e-prints, Feb. 2017.

[11] W. Atwood et al., "Pass 8: Toward the Full Realization of the Fermi-LAT Scientific Potential," 2013.

[12] K. M. Ferriere, "The interstellar environment of our galaxy," Rev. Mod. Phys., vol. 73, pp. 1031-1066, 2001.

[13] G. L. Case and D. Bhattacharya, "A new sigma-d relation and its application to the galactic supernova remnant distribution,” Astrophys. J., vol. 504, p. 761, 1998.

[14] F. Aharonian et al., "Discovery of very-high-energy $\gamma$-rays from the Galactic Centre ridge," Nature, vol. 439, pp. 695-698, Feb. 2006. 
[15] M. G. Aartsen, K. Abraham, M. Ackermann, J. Adams, J. A. Aguilar, M. Ahlers, M. Ahrens, D. Altmann, K. Andeen, T. Anderson, and et al., "All-sky Search for Time-integrated Neutrino Emission from Astrophysical Sources with 7 yr of IceCube Data," Astrophysical Journal, vol. 835, p. 151, Feb. 2017.

[16] M. G. Aartsen, M. Ackermann, J. Adams, J. A. Aguilar, M. Ahlers, M. Ahrens, D. Altmann, T. Anderson, C. Arguelles, T. C. Arlen, and et al., "Observation of High-Energy Astrophysical Neutrinos in Three Years of IceCube Data,” Physical Review Letters, vol. 113, p. 101101, Sept. 2014.

[17] GNN-web, “The Global Neutrino Network.” http://www.globalneutrinonetwork.org/, 2015.

[18] M. Ackermann et al., "Fermi-LAT Observations of the Diffuse Gamma-Ray Emission: Implications for Cosmic Rays and the Interstellar Medium," Astrophys. J., vol. 750, p. 3, 2012.

[19] K. M. Gorski, E. Hivon, A. J. Banday, B. D. Wandelt, F. K. Hansen, M. Reinecke, and M. Bartelman, "HEALPix - A Framework for high resolution discretization, and fast analysis of data distributed on the sphere," Astrophys. J., vol. 622, pp. 759-771, 2005.

[20] F. Acero et al., "Fermi Large Area Telescope Third Source Catalog,” Astrophys. J. Suppl., vol. 218, no. 2, p. 23, 2015.

[21] W. e. a. Apel, "KASCADE-Grande measurements of energy spectra for elemental groups of cosmic rays," Astropart.Phys., vol. 47, pp. 54-66, 2013.

[22] A. Albert et al., "New Constraints on all flavour Galactic diffuse neutrino emission with the ANTARES telescope," ArXiv e-prints, May 2017.

[23] S. Adrián-Martínez, M. Ageron, F. Aharonian, S. Aiello, A. Albert, F. Ameli, E. Anassontzis, M. Andre, G. Androulakis, M. Anghinolfi, and et al., "Letter of intent for KM3NeT 2.0," Journal of Physics G Nuclear Physics, vol. 43, p. 084001, Aug. 2016.

[24] G. Illuminati et al. for the ANTARES collaboration, "All-flavor Neutrino Point-like Source Search with the ANTARES Neutrino Telescope," Contribution to ICRC, 2017.

[25] A. trovato et al. for the KM3NeT collaboration, "Expectations for detection of neutrinos from point-like sources with KM3NeT/ARCA," Contribution to ICRC, 2017. 